\title{
Private Klinikketten verändern das Krankenhauswesen Chancen durch die Integration in einen Konzern
}

In vielen Branchen ist die Organisationsform des Konzerns bereits sehr erfolgreich umgesetzt. Immer öfter setzt sich auch im Krankenhauswesen die Idee eines Zusammenschlusses mehrerer Häuser unter dem Dach eines Konzerns durch. Sicherlich ist dies kein Allheilmittel für ein erfolgreiches Krankenhausmanagement, doch solche Verbundlösungen bieten viele Vorteile gegenüber kleinen kommunalen Häusern angefangen bei der Qualität der Behandlung, die unter anderem auch durch den steten Wissensaustausch innerhalb des Netzwerkes gewährleistet werden kann, bis hin zu den Sparpotenzialen, die sich aus klaren Unternehmensstrategien und dadurch transparente und optimierte Abläufe ergeben.

Krankenhausverbünde waren schon üblich, als Apotheker und Ärzte noch nicht einmal daran dachten, Filialbetriebe und Netzwerke zu gründen. Vor dem Hintergrund der neuen gesellschaftlichen und politischen Herausforderungen im Gesundheitswesen erkennen inzwischen mehr und mehr noch als Einzelbetrieb agierende Kliniken die Vorteile einer Verbundlösung. So setzt sich nicht nur der Wachstumskurs von privaten Krankenhausbetreibern fort. Immer häufiger wird auch die Zusammenführung kommunaler Häuser in einen öffentlichen Verbund propagiert. Der bisherige nachweisbare und andauernde Erfolg gibt aber vor allem der Strategie der privaten Krankenhausketten recht.

\section{Konzernstrulkturen bringen Qualitätsvorteile}

Innovative Geschäftmodelle mit wirtschaftlich sinnvollen Lösungen sind es, die sich auf dem Markt durchsetzen. Bürokratische Hürden und gesetzliche Restriktionen bremsen diese Entwicklung im Gesundheitswesen zwar oft genug, dennoch lassen sich die Argumente des Marktes nicht auf immer und ewig negieren. Neue Maßstäbe bei Preis, Qualität und Leistungsangebot können durch privatwirtschaftliche Lösungen einfacher und schneller erreicht werden.

In Deutschland allerdings nehmen die betroffenen Patienten Preis und Angebot als Aktionsparameter ihrer Ent- scheidungen nur geringfügig wahr. Dies liegt in der hohen Versorgungsdichte einerseits und der immer noch sehr weitreichenden Erstattung medizinischer Leistungen andererseits. Zweifellos misst aber jeder dem „Gut Gesundheit“ einen überdurchschnittlich hohen Stellenwert bei. Insofern ist die Qualität einer Leistung für das Nachfrageverhalten der Patienten ein besonders bedeutender Orientierungsmaßstab. Sie wird am besten über den Weg der Markenbildung kommuniziert.

Außerhalb des Gesundheitswesens ist dieses Phänomen bekannt. Mit dem Kauf eines teueren Kleidungsstückes beispielsweise verbindet man einen besonders hohen Anspruch. Nichts anderes muss im Krankenhauswesen gelingen: Das Qualitätsversprechen wird an eine Marke gebunden, die Marke wiederum mit einer hohen Qualität in Verbindung gebracht. Dieses Wechselspiel kann ein Konzern deutlich besser vermarkten als allein betriebene Häuser.

\section{Markenbildung als Schlüssel zum Erfolg}

Die Sana Kliniken gehen hier mit gutem Beispiel voran. Den eigenen Anspruch, dass die Qualität unserer Leistungen der Maßstab für das Handeln unserer Führungskräfte sein muss, wird in Zukunft ein innovativer Markenauftritt nach außen abbilden. Hierfür müssen die erstklassige Qualität der medizinischen Leistungen sowie die Kompetenzen unserer Mitarbeiter dokumentiert sein, um den Aussagen Substanz zu geben. Ein allgemeiner guter Eindruck oder ein subjektiv wahrgenommenes Image reichen nicht aus. In vielen Krankenhäusern gelingt dies durch eine Zertifizierung der Behandlungsabläufe und Managementprozesse, so auch bei Sana.

Grundsätzlich steht dieser Weg allen Häusern offen. Die Chancen für eine hochwertige Zertifizierung sind im Konzern jedoch um einiges größer als in einzelnen kommunalen Häusern. Der Wert eines Gütesiegels ergibt sich nämlich erst durch eine professionelle, einheitliche, flächendeckende und damit auch eine zentrale Vermarktung. Dieses Know how ist in Konzernen erheblich größer, da dort Erfahrungsprozesse einfließen können, die im Verbund mehrerer Einrichtungen häufiger und intensiver gemacht werden.

\section{Netzwerkeffekte dienen dem Wissensaustausch}

Die Vorteile einer Konzernstruktur sind jedoch noch weiter gefasst. In einer Zeit, in der die Halbwertszeit des Wissens gerade in der Medizin rapide sinkt, ist der Austausch von Informationen von immer größer werdender Bedeutung. Das Diagnose- und Behandlungsniveau kann nur auf einem aktuellen Stand gehalten werden, wenn dieser Wissenstransfer besonders gut gelingt.

Vernetzt ein Krankenhaus seine Leistungsträger in Medizin, Pflege und Administration besonders professionell, können exzellente Leistungs- und Lenkungsstrukturen entstehen. Dies erzeugt und fördert die Kompetenz im eigenen Konzern auf vielfältige Weise, was andere Häuser teuer hinzukaufen müssen. Dabei gilt: Der Nutzen eines Netzwerks ist umso höher, je größer es ist und je stärker es wächst. Eine breite Wissensbasis, wie sie ein Konzern bieten kann, ist somit Innovationsmotor und Wettbewerbsfaktor zugleich. 


\section{Wirtschaftliche Situation ist angespannt}

Ein hohes Qualitätsniveau und ein funktionierender Wissenstransfer sind zwar eine notwendige, aber noch keine hinreichende Bedingung für den Erfolg eines Krankenhauses. Grundlage aller Prozesse und Maßnahmen ist eine wirtschaftliche Betriebsführung. Auch hier ist der Krankenhausverbund dem Einzelbetrieb deutlich überlegen.

Die für viele Krankenhäuser bereits seit Längerem angespannte Kostensituation hat sich mit der Umsetzung der Gesundheitsreform weiter verschärft. Diese verlangt von den Häusern einen Solidarbeitrag in Höhe von 1 \% ihres Budgets zur Sanierung der Gesetzlichen Krankenversicherung. Bundesweit sind das noch einmal zirka 500 Millionen Euro pro Jahr an zusätzlichen Kosten. Hinzu kommen immense Belastungen durch die im letzten Jahr abgeschlossenen Tarifverträge für Ärzte in Höhe von schätzungsweise weiteren 1,5 Milliarden Euro.

\section{Kosteneinsparpotenziale}

Die Notwendigkeit, nach Einsparmöglichkeiten zu suchen, wird also größer und ist inzwischen für zahlreiche Einrichtungen überlebenswichtig. Durch die Zentralisierung patientenferner Dienste und die Ausgliederung von Dienstleistungen an externe Anbieter oder eigene Tochterunternehmen ist es besser möglich, sich auf das Kerngeschäft zu konzentrieren.

Private Krankenhausverbünde entwickeln schneller und nachhaltiger klare Unternehmensstrategien und sorgen so für transparente und optimale Abläufe. Damit senken sie ihre Kosten und setzten Investitionsmittel frei, die insbesondere bislang öffentlich geführte Häuser dringend benötigen. Derzeit werden bei einem Gesamtumsatz von 60 Milliarden Euro im deutschen Krankenhauswesen nur 2,7 Milliarden Euro an Investitionsmitteln von der öffentlichen Hand zugewiesen. Das ist bei gestiegenem Umsatz eine um $25 \%$ niedrigere Investitionssumme als noch 1991. Kein anderer Wirtschaftszweig in Deutschland dürfte bei Investitionen derart unterfinanziert sein. Gerade die privaten Krankenhausketten zeigen durch eine deutlich höhere Investitionsquote, dass sie die Notwendigkeit von zügig durchgeführten Ersatzund Neuinvestitionen erkannt haben, um in dem innovativen Bereich Gesund- heitswesen bestehen zu können. Private Klinikkonzerne arbeiten auch in aller Regel effizienter und sind wirtschaftlich erfolgreicher als öffentliche Häuser. Dies liegt unter anderem an der höheren Flexibilität des Managements und der strengeren betriebswirtschaftlichen Ausrichtung, was auch durch den geringeren Einfluss der Politik möglich wird. Die daraus resultierenden Vorteile werden durch die Verbundeffekte eines Konzerns verstärkt.

\section{Die Politik lässt nur langsam los}

Der Krankenhausmarkt bewegt sich. Dennoch wird die Einbindung strategischer Partner vielerorts weder diskutiert noch umgesetzt. Die Ursachen hierfür sind vielschichtig. Immer wieder wird das Argument vorgetragen, dass private Klinikketten insbesondere mit dem Mittel des Personalabbaus kurzfristig viel Geld aus den Häusern herausziehen wollen, um ihren Gesellschaftern entsprechende Dividenden zu zahlen. Die Geschichte privater Klinikketten zeigt das Gegenteil.

Nach der Integration eines Hauses in einen Konzern ist es meist nicht die „Personalschraube“, an der gedreht wird. Zunächst geht es vor allem darum, die oft unzureichende Auslastung stationärer Kapazitäten und insgesamt die Leistungszahlen auf ein zufriedenstellendes Niveau zu heben und zu stabilisieren. Für die mit diesem Prozess verbundenen Maßnahmen ist gut qualifiziertes Personal unverzichtbar. Eine Reduktion des Personals würde daher bei einer Einbindung neuer Einrichtungen in einen privaten Konzern oft kontraproduktiv sein. Voraussetzung ist natürlich, dass die Personalstruktur vor der Integration aus betriebswirtschaftlicher und medizinischer Sicht sinnvoll war.

Die politisch Verantwortlichen tun sich trotzdem häufig schwer mit der Suche nach einem strategischen Partner und der Abgabe eines Gesellschaftsanteils an einen erfolgreich arbeitenden Konzern. Die Folgen zeigen sich nur schleichend, dafür aber im Laufe der Zeit umso deutlicher. Durch das Festhalten an Liebgewonnenem versickert sehr viel Geld der öffentlichen Hand (Steuergelder!) in den Strukturen eines Systems, in dem Effizienz und Kostenbewusstsein über Jahrzehnte keine Rolle spielten.

Nach dem Ausgleich jährlicher Defizite dank stärker sprudelnder Steuereinnah- men oft in Millionenhöhe reichen die finanziellen Mittel der öffentlichen Träger dann allerdings kaum noch aus, dringend notwendige Ersatz- oder Neuinvestitionen zu subventionieren. Das Ziel der politischen Entscheidungsträger, die Versorgungssicherheit und eine hohe Qualität zu gewährleisten, wird gerade so nicht erreicht. Effiziente Strukturen eines Krankenhauskonzerns dagegen helfen, diese schwierige Aufgabe zu meistern.

\section{Privater Krankenhausverbund - ein Allheilmittel?}

Allerdings ist das Potenzial für effizientere Prozesse durch eine Verbundlösung begrenzt. Die voranschreitende Tendenz der Substitution von stationären durch ambulante Leistungen, die Herausforderungen des demografischen Wandels und des medizinischen Fortschritts sowie die immer knapper werdenden Mittel der Kostenträger bestimmen auch den Geschäftserfolg der Krankenhauskonzerne. Dies sind aber nicht die einzigen Einflussfaktoren. Leistungssteigerungs- und Kostensenkungspotenziale lassen sich durch Verbundeffekte besser und effektiver ausschöpfen. Dadurch entsteht eine nachhaltig hohe medizinische Versorgungsqualität. Private Klinikkonzerne bieten hierfür die besten Chancen.

Die Herausforderungen des Gesundheitswesens treffen auch in den kommenden Jahren insbesondere die Krankenhäuser. Nicht jedes Krankenhaus in Deutschland hat eine Legitimation zu überleben, auch nicht in einem Konzern. Die Einbindung in eine Verbundstruktur ist sicher kein Allheilmittel zur Lösung aller Probleme der Branche. Jedoch zeichnet sich derzeit eine Entwicklung deutlich ab: Das moderne und innovative Krankenhaus der Zukunft wird nicht mehr für sich alleine stehen. Der Schlüssel des Erfolgs liegt in der Integration in ein Netzwerk und oft in der Zusammenarbeit mit einem strategischen Partner.

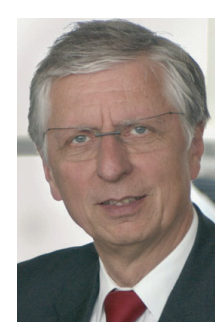

Korrespondenz

Dr. Reinhard Schwarz Sana Kliniken GmbH \& Co. KGaA Gustav-HeinemannRing 133 81739 München r.schwarz@sana.de 